[Article]

\title{
卤代苯溶剂对镓咔咯配合物光物理性质的影响：外重原子效应
}

\author{
占 轩 ${ }^{1}$ 赵 芳 $^{2}$ 张 蕾 ${ }^{2, *}$ 吕标彪 ${ }^{3} \quad$ 彭素红 $^{1}$ \\ 应 晓 ${ }^{3}$ 王 惠 ${ }^{2, *} \quad$ 刘海洋 ${ }^{1,2, *}$ \\ ('华南理工大学化学系, 广州 510641; ' 2 中山大学光电材料与技术国家重点实验室, 广州 510275; \\ 3华南理工大学应用物理系, 广州 510641)
}

\begin{abstract}
摘要: 利用紫外-可见光谱、稳态和时间分辨荧光光谱以及飞秒瞬态吸收光谱探测了不同卤代苯溶剂对三种 五氟苯基取代的镓味咯(1-Ga、2-Ga、3-Ga)光物理性质的影响, 结果表明卤代苯溶剂的色散力对于镓咔咯 电子光谱吸收峰位置的影响起着主要作用; 溶剂外重原子效应能显著降低镓咔咯的苂光量子产率。飞秒瞬态 吸收光谱表明, 光激发下, 镓咔咯与卤代苯溶剂之间可发生电子转移反应, 溶剂的重原子效应可以减缓电荷 分离态复合物电荷重组速率。
\end{abstract}

关键词: 咔咯; 镓; 卤代苯溶剂; 重原子效应; 光物理性质

中图分类号: 0641.06

\section{Influence of Halogenated Benzene Solvents on the Photophysical Properties of Gallium Corroles: the External Heavy Atom Effect}

\author{
ZHAN Xuan ${ }^{1}$
ZHAO Fang ${ }^{2}$
ZHANG Lei, ${ }^{2, *}$ YING Xiao ${ }^{3}$ \\ WANG Hui ${ }^{2, *}$ \\ LÜ Biao-Biao ${ }^{3}$ \\ LIU Hai-Yang ${ }^{1,2, *}$ \\ PENG Su-Hong ${ }^{1}$ \\ ('Department of Chemistry, South China University of Technology, Guangzhou 510641, P. R. China;
}

${ }^{2}$ State Key Laboratory of Optoelectronics Materials and Technologies, Sun-Yat Sen University, Guangzhou 510275, P. R. China;

${ }^{3}$ Department of Applied Physics, South China University of Technology, Guangzhou 510641, P. R. China)

\begin{abstract}
The photophysical properties of pentafluorophenyl-substituted gallium corroles in halogenated benzenes were investigated using ultraviolet-visible (UV-Vis), steady-state, time-resolved fluorescence, and femtosecond transient absorption spectroscopies. The results showed that the absorption maximum wavelength of the gallium corroles was mainly related to the dispersion force of the halogenated benzene solvents. The external heavy atom effect of halogenated benzenes may markedly lower the fluorescence quantum yield of gallium corrole complexes. Photoinduced electron transfer between the gallium corroles and halogenated benzene solvents was detected by femtosecond transient absorption spectroscopy. The experimental evidence showed that the heavy atom effect of the solvent might lower the charge recombination rate of charge-separated gallium corrole-solvent complexes.
\end{abstract}

Key Words: Corrole; Gallium; Halogenated benzene solvent; External heavy atom effect; Photophysical property

Received: November 3, 2015; Revised: December 31, 2015; Published on Web: January 4, 2016.

*Corresponding authors. LIU Hai-Yang, Email: chhyliu@scut.edu.cn; Tel: +86-20-22236805. WANG Hui, Email: stswh@mail.sysu.edu.cn; Tel: +86-20-84110972. ZHANG Lei, Email: zhlei28@mail.sysu.edu.cn; Tel: +86-20-84110972.

The project was supported by the National Natural Science Foundation of China (21171057, 21371059), National Key Basic Research Program of China (973) (2013CB922403), and Open Fund of State Key Laboratory of Opto-Electronic Material and Technologies of SunYat-Sen University, China (OEMT-2015-KF-05).

国家自然科学基金(21171057, 21371059), 国家重点基础研究发展规划项目(973) (2013CB922403)和中山大学光电材料与技术国家重点实验室 开放基金(OEMT-2015-KF-05)资助项目

(C) Editorial office of Acta Physico-Chimica Sinica 


\section{1 引言}

咔咯配合物已成为卟啉化学的热点研究课 题 ${ }^{1,2}$ 。由于咔咯大环 “一锅” 合成法 ${ }^{3,4}$ 的发现使得 其在催化 ${ }^{5}$, 光化学 ${ }^{6}$, 生物化学 ${ }^{7,8}$ 和配位化学 ${ }^{9,10}$ 等 领域得到广泛应用。金属镓咔咯配合物具有较好 的双光子效应、较高的 $S_{1}-S_{0}$ 苂光量子产率以及在 含氧条件下的高的单线态氧产率, 这些特性使其 在光动力治疗 ${ }^{11}$ 、染料敏化太阳能电池 ${ }^{12,13}$ 以及苂光 探针 ${ }^{14}$ 方面具有潜在的应用前景。镓咔咯配合物的 生物无机化学研究已经取得了相当的进展。水溶 性镓磺酸基咔咯与特定蛋白结合后能靶向识别并 杀死癌细胞 ${ }^{15-17} ; \beta$-位碘代的镓磺酸基咔咯配合物 具有很长的三重态寿命, 在光动力治疗中有潜在 的应用。咔咯配合物作为一类潜在的光动力治疗 (PDT) 光敏剂, 溶剂对其光物理性质的影响是个重 要的课题。目前, 有关溶剂效应对于镓咔咯光物 理性质影响的文献还较少。早前我们组 ${ }^{18}$ 曾报道过 溶剂极性对于镓咔咯光物理性质的影响。本文利 用卤代苯为介质, 以三种具有不同推拉电子性质 的镓咔咯配合物 (图 1)为模型, 考查了溶剂的外重 原子效应对镓咔咯配合物的紫外吸收光谱、荧光 光谱、苂光量子产率以及苂光寿命等光物理性质 的影响, 特别是测定了不同卤代苯溶剂中 $3-\mathrm{Ga}$ 的 飞秒瞬态吸收光谱, 探讨了外重原子效应对于其 激发态吸收的影响。

\section{2 实验部分}

\section{1 仪器与试剂}

\section{1 .1 仪 器}

U-3900 型紫外-可见吸收光谱仪(日本日立公司 生产); F-4500 型荧光光谱仪 (日本日立公司生 产); FLSP920 组合式苂光寿命与稳态苂光光谱仪
(德国 Bruker); AVANCE Digital $400 \mathrm{MHz}$ 超导核磁 共振谱仪(德国 Bruker), 氛代三氯甲烷 $\left(\mathrm{CDCl}_{3}\right)$ 为溶 剂, 四甲基硅烷(TMS)为内标。飞秒瞬态吸收仪器 的搭建及使用见文献 ${ }^{19}$, 该光路系统所使用的光源 是重复频率为 $500 \mathrm{~Hz}$ 的钛宝石再生放大器激光器 (35 fs, $800 \mathrm{~nm}$ )。基频光被光稧分为两束, 一束能 量约为 $6 \mu \mathrm{J} \cdot$ pulse $^{-1}$ 被聚集至流动水中产生超连续 白光, 作为探测光。另一束通过偏嗍酸钡晶体 (BBO)倍频晶体产生 $400 \mathrm{~nm}$ 的倍频光, 作为洜浦 光。泵浦光的脉冲通过光纤光谱仪 (Avantes, AvaSpec_ULS2048L-USB2)记录, 将洜浦光的偏振 方向与探测白光偏振方向调至 $54.7^{\circ}$, 最终泵浦光 能量约为 $2 \mu \mathrm{J} \cdot$ pulse $^{-1}$, 时间分辨率为 $35 \mathrm{fs}$ 。

\subsection{2 试 剂}

无机试剂: 氯化镓( $>99 \%$, sigma 公司)。

有机试剂: 吡咯 $(\geq 98 \%) ; 2,3$-二氯-5,6-二氧 基-1,4-苯( $\geq 98 \%$, Acros Oranics 公司); 苯甲醛( $\geq$ $98 \%$, 广州试剂公司); 苯(分析纯, 广州试剂公 司); 甲苯(分析纯, 广州试剂厂); 氯苯(分析纯, 广州试剂厂); 乙醇(分析纯, 广州试剂厂); 吡啶 (分析纯, 广州试剂厂); 二氯甲烷(DCM) (分析 纯, 广州试剂厂); 三氟乙酸(TFA) (分析纯, 广州 试剂厂); 三乙胺(分析纯, 广州试剂厂); 正己烷 (分析纯, 广州试剂厂); 溴苯( $>98 \%$, 国药集团化 学试剂公司); 氟苯(>98\%, Adamsa peagent 公 司); 碘苯( $>98 \%$, Adamsa peagent公司)。

\section{2 样品的制备及表征}

目标化合物的合成和表征见我们组相关文 献 ${ }^{18}$ 。

\section{3 结果与讨论}

\section{1 紫外-可见光谱}
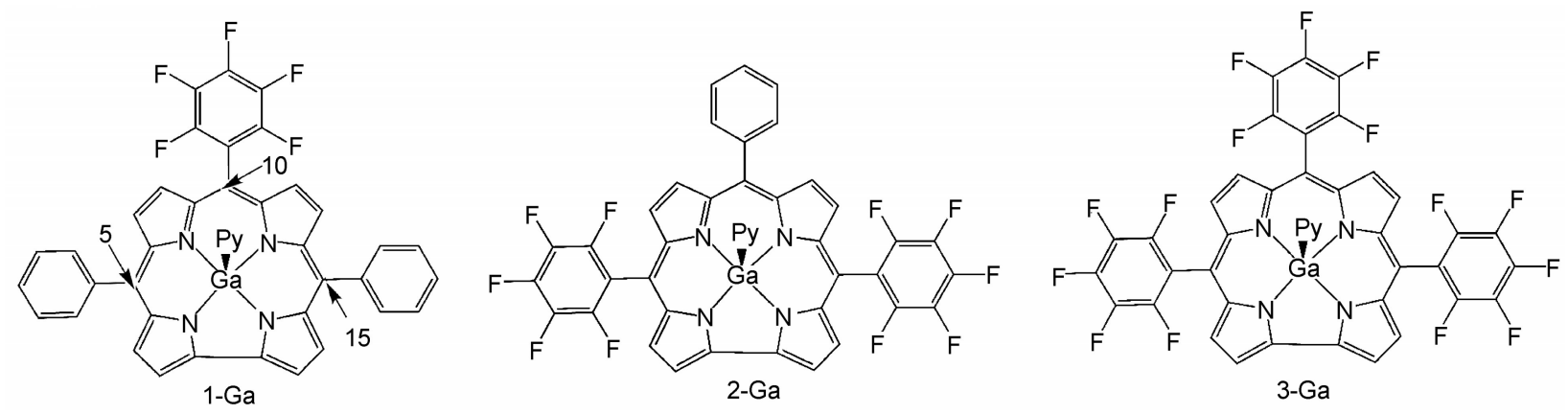

图 1 镓咔咯配合物的分子结构

Fig.1 Molecular structures of gallium corrole complexes 
三种镓咔咯在不同卤代苯溶剂中的紫外-可见 光谱数据列于表 1 。图 2 给出了 $1-G a$ 的电子光谱, 在不同卤代苯溶剂中，镓咔咯配合物均显示特征 的电子光谱, 在 $400 \mathrm{~nm}$ 左右较尖锐的 Soret 带和 500-650 nm 处强度较小的 $Q$ 带。在不同的卤代苯 溶剂环境下, 可以发现 Soret 带与 $Q$ 带的吸收强度 均有不同程度的减弱, 以 $1-\mathrm{Ga}$ 为例, 从氟苯到碘 苯溶剂, Soret带有较小的红移(422-426 nm)。在 同种卤代苯溶剂中, 2-Ga 与 3-Ga 的 Soret 带吸收峰 位置基本相似, 但均较 1-Ga 蓝移, 这是因为在中 位苯环上引入吸电子基团会增加 Corrole 的 HOMOLUMO 能隙 ${ }^{20}$ 。

非极性溶剂对溶质电子光谱吸收峰位置的影 响, 可以用如下公式描述表示 ${ }^{21}$ :

$$
v_{\mathrm{A}}^{\mathrm{S}}-v_{\mathrm{A}}^{\mathrm{G}}=A \times F(n)
$$

表 1 镓咔咯在不同卤代苯溶剂中的紫外可见谱数据

Table $1 \mathrm{UV}$-Vis spectral data of gallium corroles in different halogenated benzene solvents

\begin{tabular}{cccc}
\hline \multirow{2}{*}{ Gallium corrole } & Solvent & \multicolumn{2}{c}{$\lambda / \mathrm{nm}$} \\
\cline { 3 - 4 } 1-Ga & fluorobenzene & 422 & $Q$ band \\
\cline { 3 - 4 } & chlorobenzene & 424 & 567,600 \\
& bromobenzene & 425 & 567,603 \\
\multirow{2}{*}{$2-\mathrm{Ga}$} & iodobenzene & 426 & 570,603 \\
& fluorobenzene & 420 & 574,600 \\
& chlorobenzene & 423 & 575,601 \\
& bromobenzene & 424 & 575,602 \\
\multirow{2}{*}{$3-\mathrm{Ga}$} & iodobenzene & 425 & 576,604 \\
& fluorobenzene & 420 & 571,594 \\
& chlorobenzene & 422 & 573,596 \\
& bromobenzene & 423 & 573,597 \\
& iodobenzene & 425 & 573,597 \\
\hline
\end{tabular}

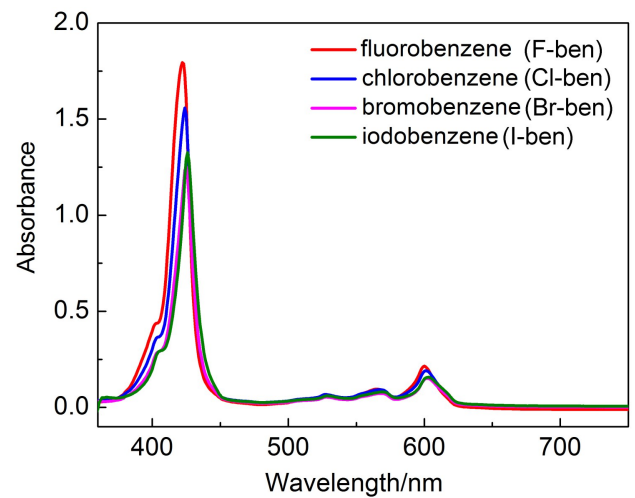

图 2 不同卤代苯溶剂中 1-Ga的紫外-可见光谱

Fig.2 UV-Vis spectra of 1-Ga in different halogenated benzene solvents color on web version
$V_{\mathrm{A}}^{\mathrm{G}}\left(\mathrm{cm}^{-1}\right)$ 表示气态样品的最大电子吸收峰的频率, 对于固定样品为固定值： $v_{\mathrm{A}}^{\mathrm{S}}\left(\mathrm{cm}^{-1}\right)$ 表示样品在溶剂 中的最大电子吸收峰的频率, 其中 $F(n)=$ $\frac{n^{2}-1}{2 n^{2}+1} ; n$ 为溶剂的折射率, $A$ 是与溶质本身性质 有关的参数。镓咔咯在不同溶剂中的 Soret 带吸收 $\left(v_{\mathrm{A}}\right)$ 为纵坐标, 以溶剂的 $F(n)$ 值为横坐标作图, 发 现其良好的线性关系(见图 3)。说明色散力对于镓 咔咯电子光谱的影响占主要作用 ${ }^{21}$ 。当考虑溶剂极 性效应时，可以用 $F(\varepsilon, n)$ 代替 $F(n), F(\varepsilon, n)$ 计算公 式如下 ${ }^{22}$ :

$$
F(\varepsilon, n)=\frac{\varepsilon-1}{\varepsilon+2}-\frac{n^{2}-1}{n^{2}+2}
$$
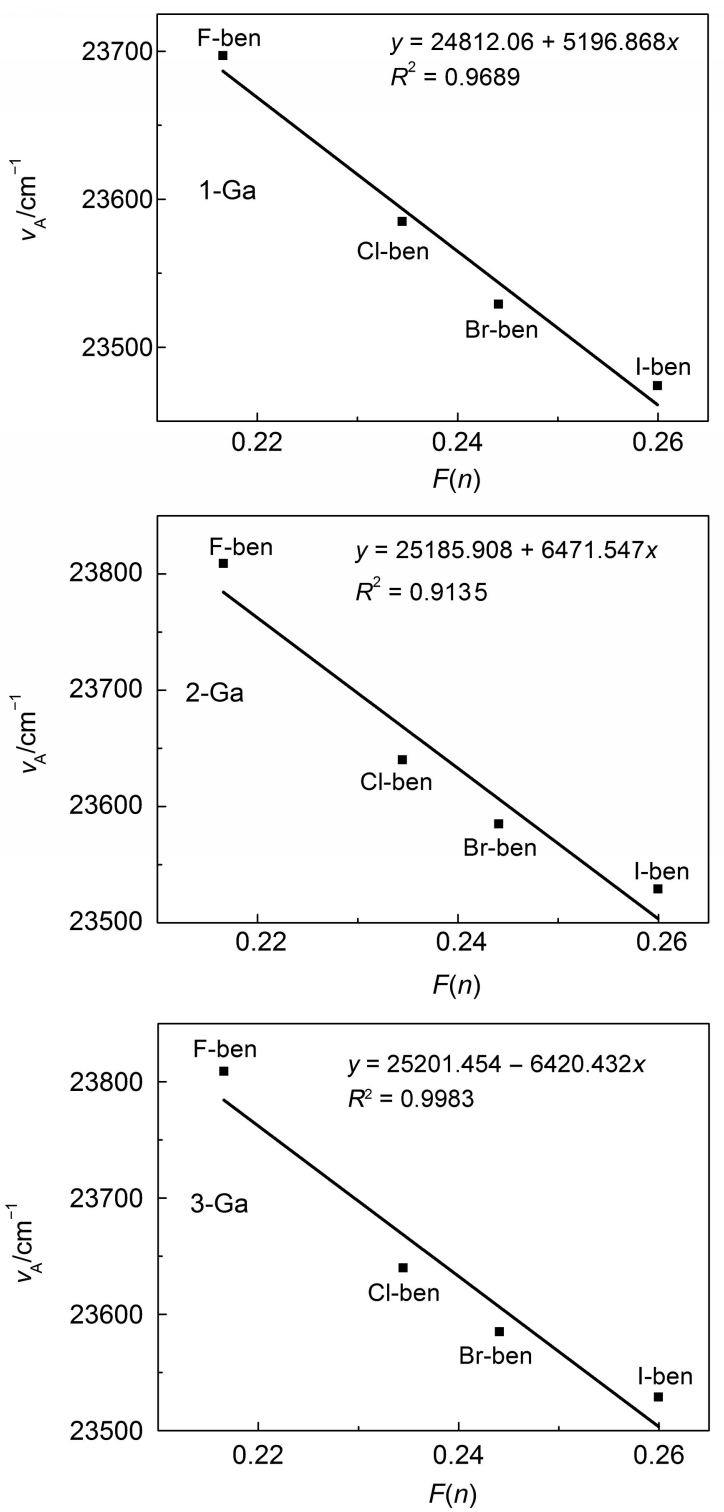

图 3 镓咔咯的 Soret 带吸收峰对卤代苯 $F(n)$ 函数作图

Fig.3 Plot of Soret band of gallium corroles $v$ s function $F(n)$ of halogentaed-benzene solvents 
发现 $v_{\mathrm{A}}-F(\varepsilon, n)$ 线性关系不好(图 4), 说明卤代苯溶 剂的偶极作用对镓咔咯电子光谱影响较小 ${ }^{21}$ 。

\section{2 荧光光谱}

镓咔咯化合物具有典型的双苂光性质 ${ }^{23}$, 即不 仅存在 $S_{1} \rightarrow S_{0}$ 的苂光, 还可以通过苂光上转换的 技术手段将红外波段信号的测量转变为可见波段 信号的测量从而检测到其 $S_{2} \rightarrow S_{0}$ 的苂光信号。图 5 为三种镓咔咯在四种卤代苯溶剂中的荧光发射光 谱, 镓咔咯化合物在卤代苯溶剂中的发射峰位置 均在 $600 \mathrm{~nm}$ 左右, 在同一溶剂中, 随着 meso 位取 代基 $\mathrm{F}$ 原子的增多, 咔咯环上电子密度减小, 造成 分子基态与激发态之间能隙增大使发射峰出现蓝
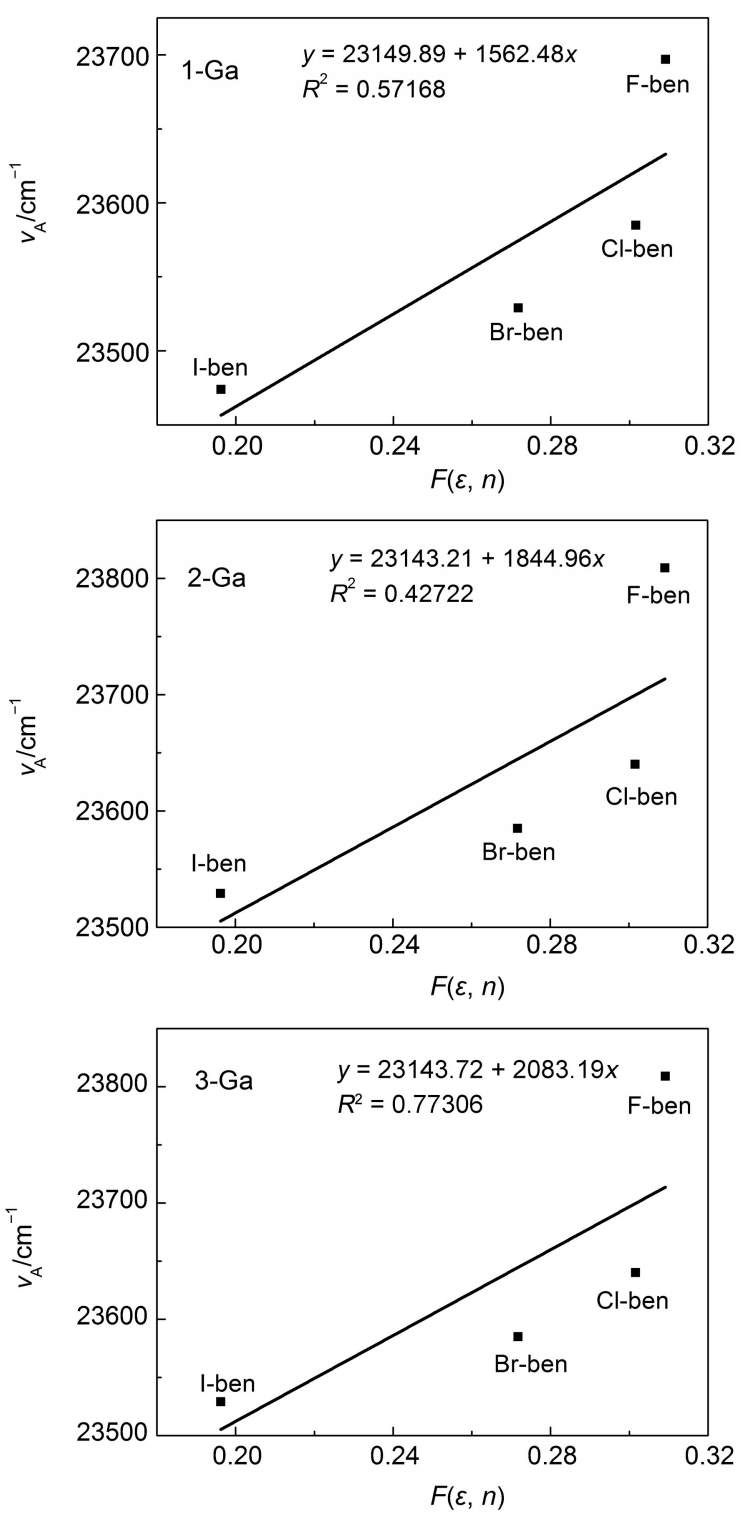

图 4 镓咔咯的Soret 带吸收峰对卤代苯 $F(\varepsilon, n)$ 函数作图 Fig.4 Plot of Soret band of gallium corroles $v$ s function $F(\varepsilon, n)$ of halogentaed-benzene solvents
移 $^{18}$ 。以在氟苯中为例(图 5(d)), 1-Ga、2-Ga、3$\mathrm{Ga}$ 的发射峰位置分别为 $610 、 608 、 598 \mathrm{~nm}$ 。与此 同时, $1-\mathrm{Ga}$ 的苂光强度最大, 而 $2-\mathrm{Ga}$ 与 $3-\mathrm{Ga}$ 的苂 光强度较小, 这可能是因为 $1-\mathrm{Ga}$ 环内电子密度的 分布更加不均, 从而有效促进其苂光过程 ${ }^{24}$ 。而对 于 2-Ga 与 3-Ga而言，5、15 位处的五氟苯基是相 对称的, $\mathrm{F}$ 原子吸电子导致环内电子密度的分布更 均匀, 从而有利于非辐射跃迁过程导致其荧光强 度比 1-Ga 的弱。

通过图 5(a-c) 可以看出, 镓咔咯的苂光强度从 氟苯到碘苯溶剂呈明显的下降趋势, 这是因为激 发态产生苂光的辐射过程与其系间窝越的非辐射 过程是彼此竞争的过程, 溶剂的外重原子效应能 够有效地增强 $S_{n} \rightarrow T_{n}$ 的系间窝越过程, 导致荧光 强度的降低。苂光量子产率的计算可以用以下公 式进行计算 ${ }^{25}$ :

$$
\Phi_{\mathrm{u}}=\Phi_{\mathrm{s}} \times\left(F_{\mathrm{u}} / F_{\mathrm{s}}\right) \times\left(A_{\mathrm{s}} / A_{\mathrm{u}}\right) \times\left(n_{\mathrm{s}}\right)^{2} \div\left(n_{\mathrm{u}}\right)^{2}
$$

式中 $\Phi_{\mathrm{u}} 、 F_{\mathrm{u}} 、 A_{\mathrm{u}} 、 n_{\mathrm{u}}$ 分别表示待测样品在特定溶剂 中的苂光量子产率、荧光激发强度、激发波长下 的吸光度以及溶剂的折射率。 $\Phi_{\mathrm{s}} 、 F_{\mathrm{s}} 、 A_{\mathrm{s}} 、 n_{\mathrm{s}}$ 为参 考样品四苯基卟啉 $\left(\mathrm{H}_{2} \mathrm{TPP}\right)$ 在甲苯中的苂光量子产 率 $(0.11)^{26}$ 、荧光激发强度、激发波长下的吸光度以 及甲苯的折射率(1.4961)。其相应计算结果列于表 2。由于碘苯有最大的重原子碘, 所有镓咔咯的苂 光量子产率在碘苯中均急剧下降。

为了更好地分析卤代苯溶剂对于镓咔咯化合 物的激发态性质的影响, 我们利用组合式苂光光 谱仪测定镓咔咯化合物在不同芳香溶剂下的苂光 寿命, 相关数据列于表 2 。图 6 给出 $1-\mathrm{Ga}$ 在四种卤 代苯溶剂中的荧光衰减曲线, 在氟苯及氯苯中均 采用单指数拟合且契合程度好, 而在溴苯以及碘 苯中采用双指数拟合使得偏离程度较小, 将拟合 出的寿命的主要成分 $\tau_{1}$ 归为荧光寿命, 而 $\tau_{2}$ 组分可 能是激发态的互变异构体或电荷分离态。对照表 2 中 2-Ga 与 $3-\mathrm{Ga}$ 的苂光寿命数据可得, 由于系间窝 越过程与苂光过程是彼此竞争的过程, 故随着外 重原子效应的加强, 分子内系间窝越过程的加剧 使得苂光过程相应减弱 ${ }^{27}$ 并造成苂光寿命的减少, 这与表 2 中苂光寿命数据大致的下降趋势是一致 的, 但对于 $1-\mathrm{Ga}$ 在溴苯中较长的荧光寿命, 尚不 明确其原因。对于 3-Ga, 在碘苯溶剂中由于极弱 的苂光强度无法测到其荧光寿命。

利用苂光寿命和镓咔咯在卤代苯溶剂中的荧 

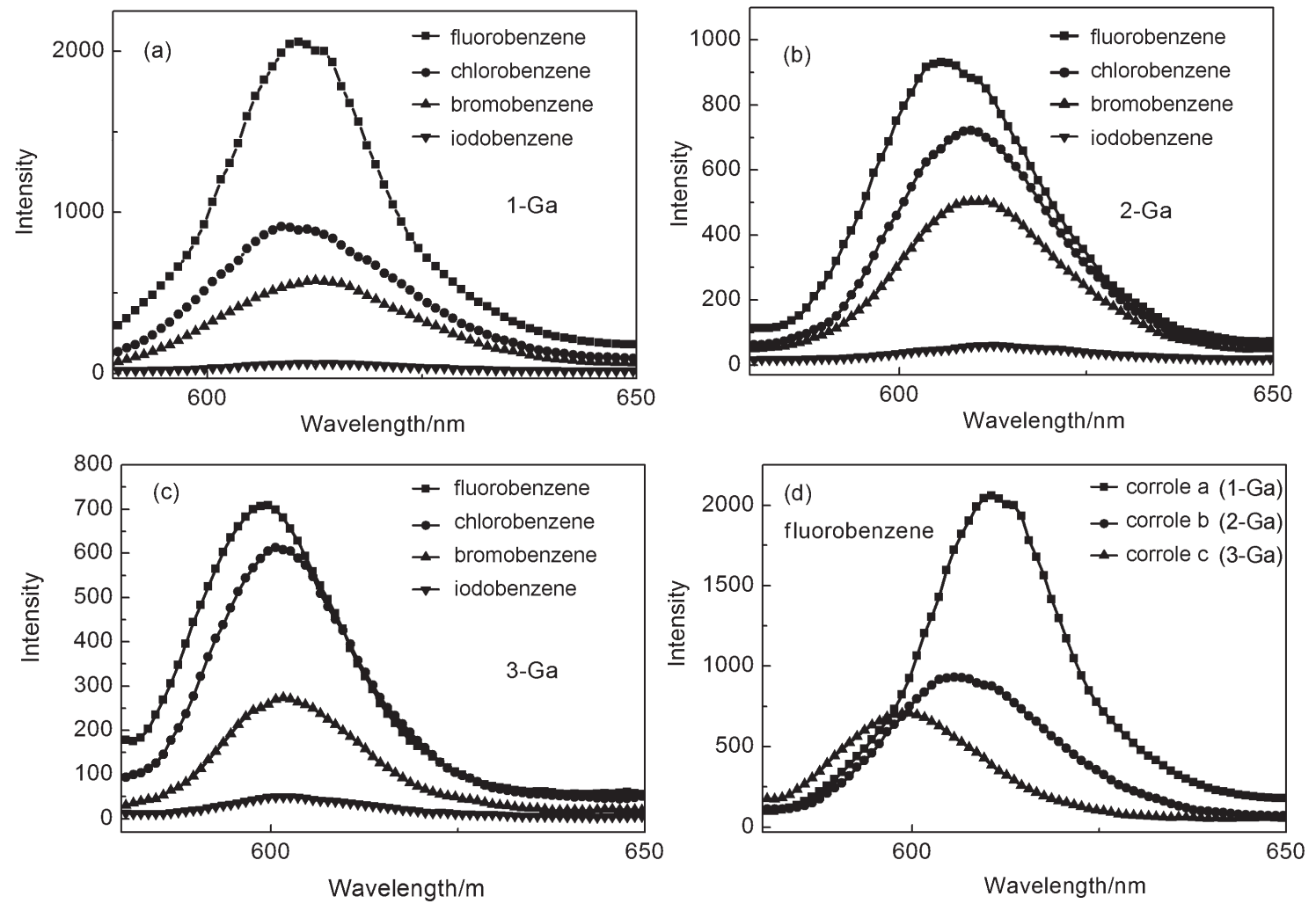

图 5 镓咔咯在不同溶剂 $(a-c)$ 与在氟苯 $(d)$ 中的荧光发射光谱

Fig.5 Fluorescence emission spectra of gallium corroles in different solvents $(\mathrm{a}-\mathrm{c})$ and fluorobenzene (d)

表 2 镓咔咯在卤代苯溶剂中的光物理性质数据

Table 2 Photophysical data of gallium corroles in halogenated benzene solvents

\begin{tabular}{ccccccc}
\hline Sample & Solvent & $n$ & $\Phi^{\mathrm{a}}$ & $\tau^{\mathrm{b} / \mathrm{ns}}$ & $10^{-8} K_{\mathrm{r}}^{\mathrm{c}} / \mathrm{s}^{-1}$ & $10^{-8} K_{\mathrm{rr}}^{\mathrm{d}} / \mathrm{s}^{-1}$ \\
\hline \multirow{2}{*}{-Ga } & fluorobenzene & 1.4650 & $0.5264 \pm 0.0060$ & $2.700 \pm 0.016$ & $1.940 \pm 0.020$ & $1.75 \pm 0.02$ \\
& chlorobenzene & 1.5246 & $0.2877 \pm 0.0030$ & $2.500 \pm 0.013$ & $1.150 \pm 0.030$ & $2.85 \pm 0.03$ \\
& bromobenzene & 1.5590 & $0.2500 \pm 0.0030$ & $3.100 \pm 0.012$ & $0.800 \pm 0.010$ & $2.42 \pm 0.02$ \\
& iodobenzene & 1.6200 & $0.0314 \pm 0.0040$ & $2.300 \pm 0.013$ & $0.136 \pm 0.010$ & $4.20 \pm 0.03$ \\
\multirow{3}{*}{-Ga } & fluorobenzene & 1.4650 & $0.480 \pm 0.005$ & $2.400 \pm 0.011$ & $2.00 \pm 0.03$ & $2.17 \pm 0.02$ \\
& chlorobenzene & 1.5246 & $0.260 \pm 0.003$ & $2.300 \pm 0.012$ & $1.13 \pm 0.02$ & $3.22 \pm 0.05$ \\
& bromobenzene & 1.5590 & $0.174 \pm 0.002$ & $2.200 \pm 0.012$ & $0.79 \pm 0.01$ & $3.75 \pm 0.04$ \\
& iodobenzene & 1.6200 & $0.056 \pm 0.001$ & $1.800 \pm 0.011$ & $0.31 \pm 0.01$ & $5.24 \pm 0.03$ \\
$3-G a$ & fluorobenzene & 1.4650 & $0.2710 \pm 0.0030$ & $1.300 \pm 0.011$ & $2.08 \pm 0.02$ & $5.60 \pm 0.04$ \\
& chlorobenzene & 1.5246 & $0.3130 \pm 0.0040$ & $2.500 \pm 0.012$ & $1.25 \pm 0.02$ & $2.75 \pm 0.02$ \\
& bromobenzene & 1.5590 & $0.1520 \pm 0.0020$ & $1.200 \pm 0.010$ & $1.25 \pm 0.01$ & $7.07 \pm 0.05$ \\
& iodobenzene & 1.6200 & $0.0277 \pm 0.0020$ & - & - & - \\
\hline
\end{tabular}

${ }^{a}$ fluorescence quantum yield \pm standard uncertainty; ${ }^{b}$ measured fluorescence lifetime \pm standard uncertainty; ${ }^{c}$ calculated radiative transition constant \pm standard uncertainty; ${ }^{\mathrm{d}}$ calculated irradiative transition constant \pm standard uncertainty

光量子产率数据, 可以计算其辐射跃迁常数 $K_{\mathrm{r}}$ $\left(K_{\mathrm{r}}=\varphi_{\mathrm{u}} / \tau_{\mathrm{f}}, \varphi_{\mathrm{u}}\right.$ 为苂光量子产率, $\tau_{\mathrm{f}}$ 为荧光寿命 $)$ 及非 辐射跃迁常数 $K_{\mathrm{nr}}\left(K_{\mathrm{nr}}=\left(1-\varphi_{\mathrm{u}}\right) / \tau_{\mathrm{f}}\right)($ 表 2$)$ 。由于外重 原子效应促进了系间窝越这种非辐射跃迁，三种 镓咔咯在氟苯中的 $K_{\mathrm{r}}$ 数值最大, 在碘苯中的 $K_{\mathrm{nr}}$ 数 值最大，这与所预期的结果相同。镓咔咯在氟苯 中的荧光强度和苂光量子产率都较之在其他卤代
苯溶剂中高, 这不仅是因为氟原子较弱的重原子 性, 还跟其很高的电负性以及较小的范德华半径 有关，相比于其他卤素原子而言更能提高苂光的 稳定性 ${ }^{28}$ 。有文献曾报道过卤代苯溶剂对于卟吩苂 光的猝灭机理 ${ }^{29}$, 一般认为有两种方式: 重原子溶 剂分子与激发态溶质分子发生碰撞时, 通过电子 交换机理对激发态的苂光过程产生作用, 这种方 

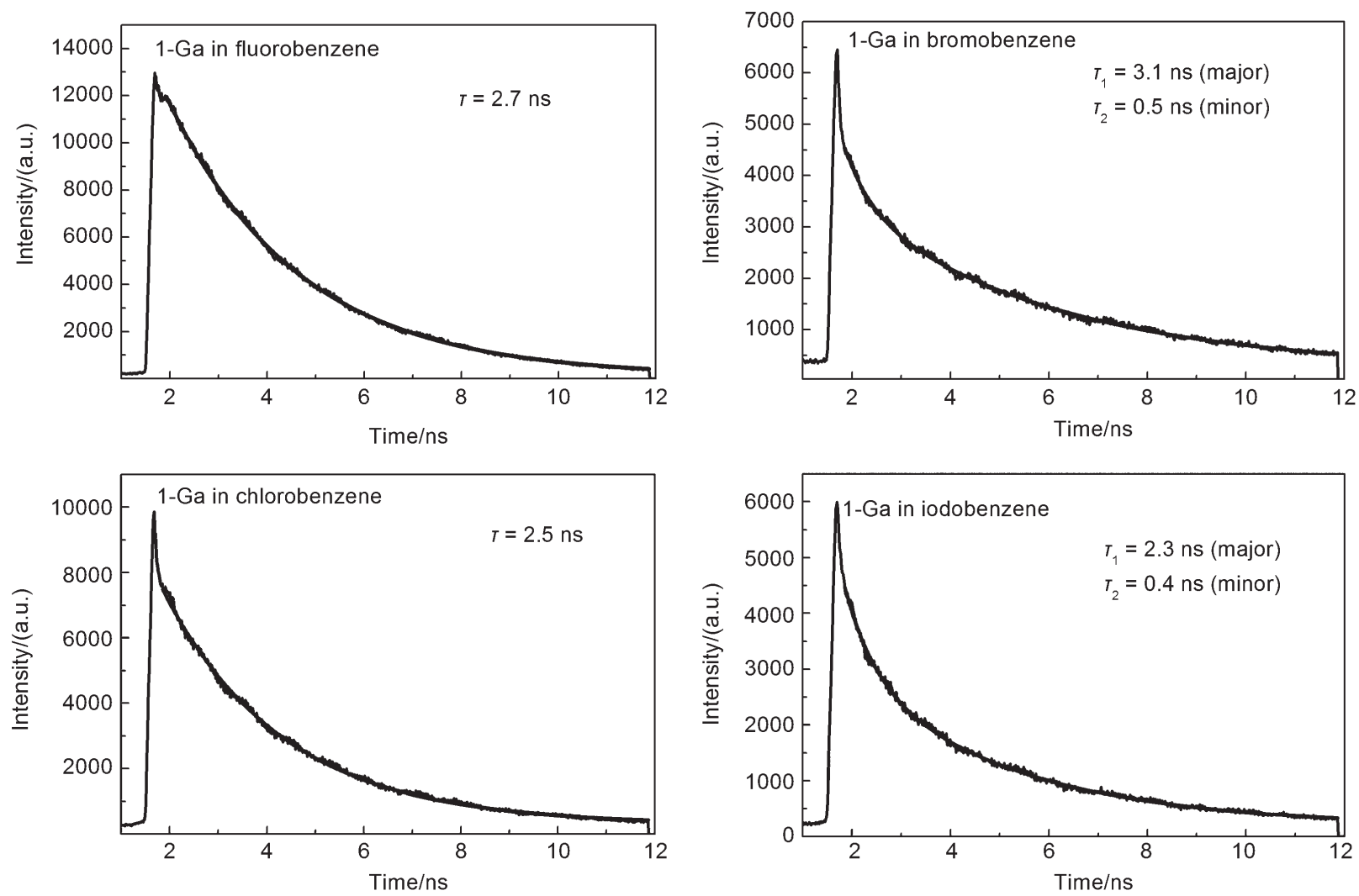

图 6 卤代苯溶剂中 1-Ga 的荧光衰减曲线

Fig.6 Fluorescence decay curves of 1-Ga in halogenated solvents

$\lambda_{\text {excitation }}=405 \mathrm{~nm}$

式称之为动态猝灭; 重原子溶剂分子与激发态分 子产生电荷转移复合物, 这种方式称之为静态猝 灭。一般认为, 卤代苯溶剂猝灭苂光是通过产生 电荷转移复合物的方式进行的, 溶剂的重原子可 以通过旋一轨偶合增大单重态到三重态的系间窝越 过程, 与卤素取代咔咯的分子内重原子效应类 似 ${ }^{30}$ 。

\section{3 瞬态吸收光谱}

为考查卤代本溶剂对镓咔咯配合物激发态 $S_{2}-S_{1}$ 内转换及其振动弛豫性质的影响, 本文测定了 3$\mathrm{Ga}$ 在不同卤代苯溶剂中的超快速瞬态吸收光谱(图 7)。结合稳态紫外可见吸收光谱与苂光光谱, 3-Ga 在氟苯中最大瞬态吸收峰位于 $448 \mathrm{~nm}$ 处强的正吸 收信号归属为 3-Ga 的激发态吸收(ESA)信号, 433 $\mathrm{nm}$ 处弱的负吸收为 Soret 带的基态漂白(GB)信号。 考虑到 3-Ga 在的荧光发射峰在 $600 \mathrm{~nm}$ 左右, 596 $\mathrm{nm}$ 处较强的负瞬态吸收峰可以归属为 $Q$ 带的基态 漂白和 $\mathrm{S}_{1}$ 态的受激发射(SE) 重叠信号。此外, 在波 长 $700 \mathrm{~nm}$ 处还有一个明显的 ESA 信号。在不同卤 代苯溶剂中, 3-Ga 的瞬态吸收光谱明显不同。最
具特征的变化是随着卤素原子量的增加波长在 450-500 nm 处 ESA 的峰变宽, $600 \mathrm{~nm}$ 处的负吸收 峰减弱。在碘苯溶剂中, 3-Ga 的苂光最弱, 瞬态 吸收光谱中 $600 \mathrm{~nm}$ 处的负峰则基本消失, 表明该 峰主要来源于 $S_{1}$ 态的受激发射, 而 $Q$ 带的基态漂白 所占的成分很小。

延迟时间在约 3-70 ps 时, 瞬态吸收 $\Delta \mathrm{OD}$ 值变 化较快, 而在约 70-1000 ps 时, $\Delta \mathrm{OD}$ 值变化放缓 (图 7), 显示有多种激发态物种存在。图 8 给出 3$\mathrm{Ga}$ 在各卤代苯溶剂中的最大激发态吸收峰处信号 的动力学曲线, 衰减信号符合三指数拟合, 得到 寿命分别为 $\tau_{\mathrm{d} 1} 、 \tau_{\mathrm{d} 2} 、 \tau_{\mathrm{d} 3}$ 的三种瞬态物种。其中 $\tau_{\mathrm{d} 3}$ 在 纳秒数量级, 与荧光寿命接近, 它对应于 $S_{1}$ 态物种 的荧光和系间窝越过程。不同卤代苯溶剂中 $3-\mathrm{Ga}$ 的 $\tau_{\mathrm{d} 2}$ 的变化范围较大, 在 17-110 ps 之间。一些自 由咔咯的 $S_{1}{ }^{*} \rightarrow S_{1}$ 振动弛豫在 16-109 ps 之间 ${ }^{19}, \tau_{\mathrm{d} 2}$ 可以归属为 3-Ga 的 $S_{1}{ }^{*} \rightarrow S_{1}$ 振动弛豫过程。3-Ga 的 $\tau_{\mathrm{d} 1}$ 时尺度比较短, 卤代苯溶剂中可观察到其在 0.77-8.64 ps。Steer 等 ${ }^{23}$ 曾研究过 3-Ga 在甲苯溶剂 中 $S_{2}$ 态的性质, 测得其 $S_{2}$ 态的寿命为 $0.28 \mathrm{ps}$ 。与 $3-$ 

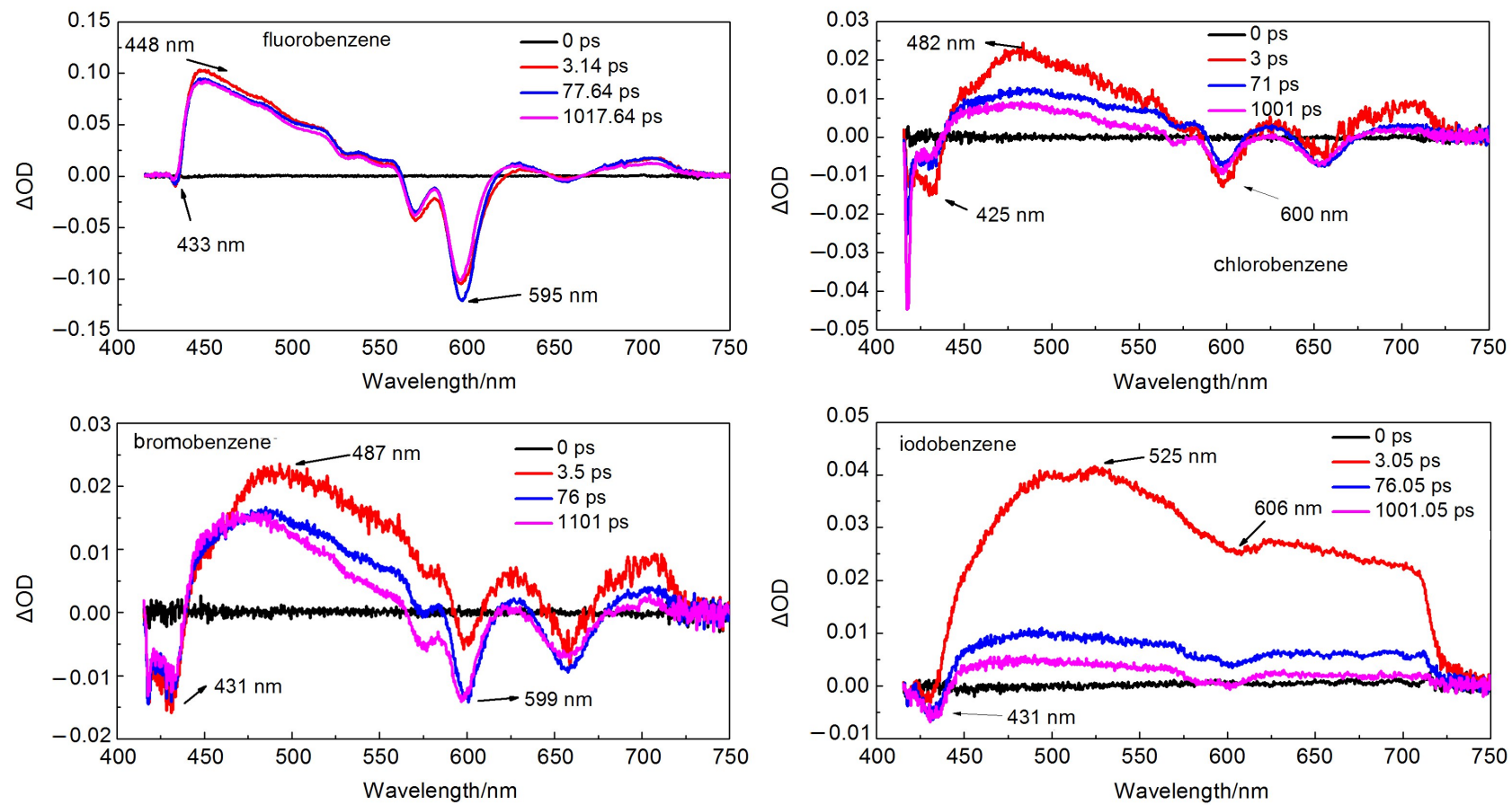

图 7 卤代苯溶剂中 3-Ga的飞秒瞬态吸收谱图

Fig.7 Femtosecond transient absorption spectra of 3-Ga in halogenated-benzene solvents excitation at $400 \mathrm{~nm}$, color on web version
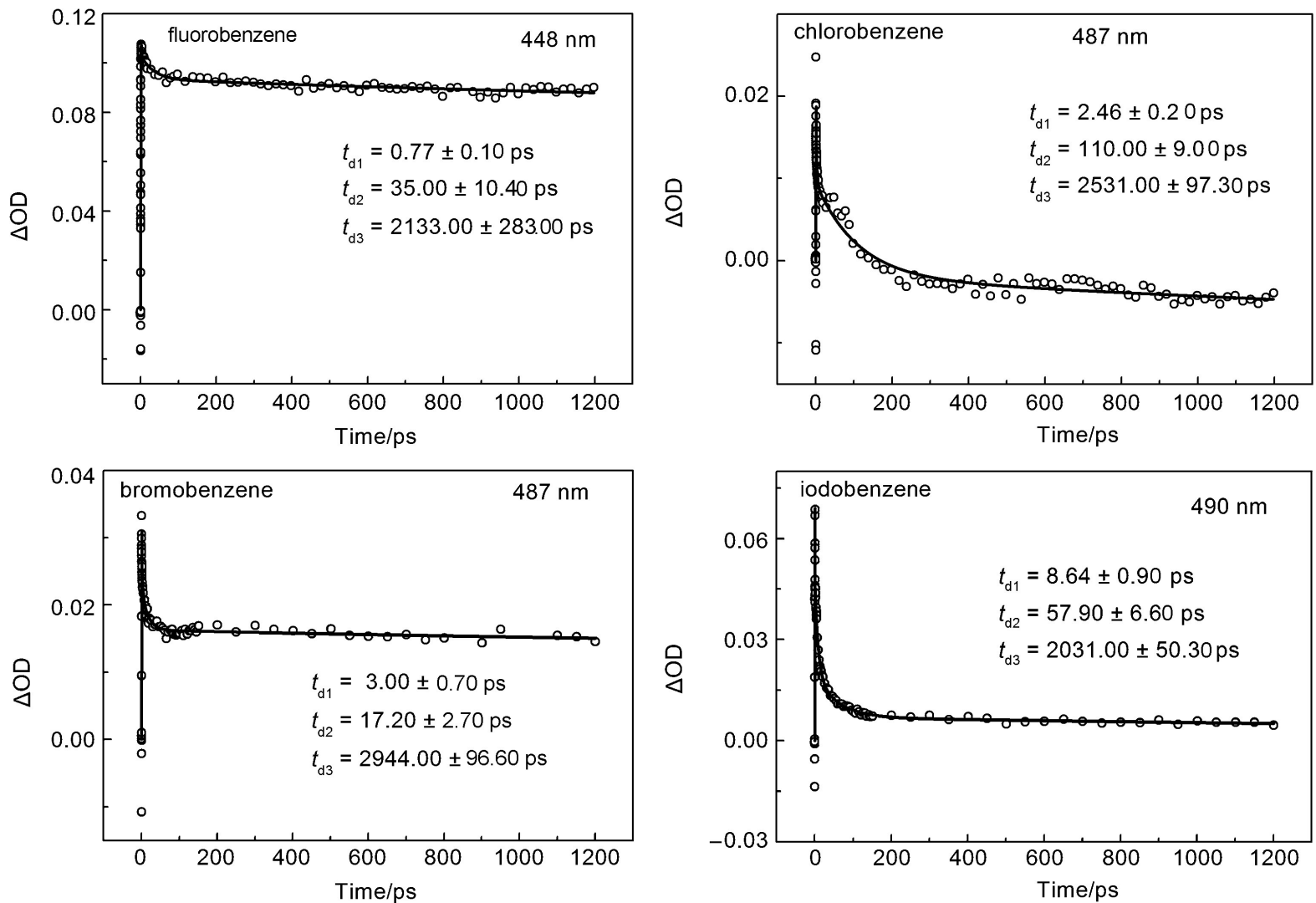

图 8 卤代苯溶剂中 3-Ga最强激发态吸收峰信号的时间曲线

Fig.8 Time profiles at the maximum wavelengths of excited state absorption peak for 3-Ga in halogenated-benzene solvents 
$\mathrm{Ga}$ 对应的自由咔咯 3 在甲苯溶液中 $S_{2} \rightarrow S_{1}{ }^{*}$ 内转换 过程的寿命为 $0.62 \mathrm{ps}^{19}$ 。因此, 3-Ga在卤代苯溶剂 中的 $\tau_{\mathrm{d} 1}$ 对应的是 $S_{2} \rightarrow S_{1}$ *内转换过程。

有趣的是 3-Ga在卤代苯溶剂中, $S_{2} \rightarrow S_{1}$ 寿命 显著延长, 在碘苯中达到了 $8.64 \mathrm{ps}$ 。这是由于 3$\mathrm{Ga}$ 与卤代苯溶剂分子之间发生了光诱导电子转移 (ET)形成了激发态电荷分离 $(\mathrm{CS})$ 复合物。CS 复合 物形成的另一个证据是 3-Ga在氯苯、溴苯与碘苯 溶剂中 450-500 nm 处 ESA 的峰显著变宽, 这一现 象在锌卟啉体系中也可以观察到 31,32 。由于激发 CS 态的形成是个超快速过程 ${ }^{32}$, 在卤代苯溶剂中 3-Ga 的 $\tau_{\mathrm{d} 1}$ 反映的是 $S_{2 \mathrm{CS}} \rightarrow S_{1}$ *过程, 可近似拆分为电荷

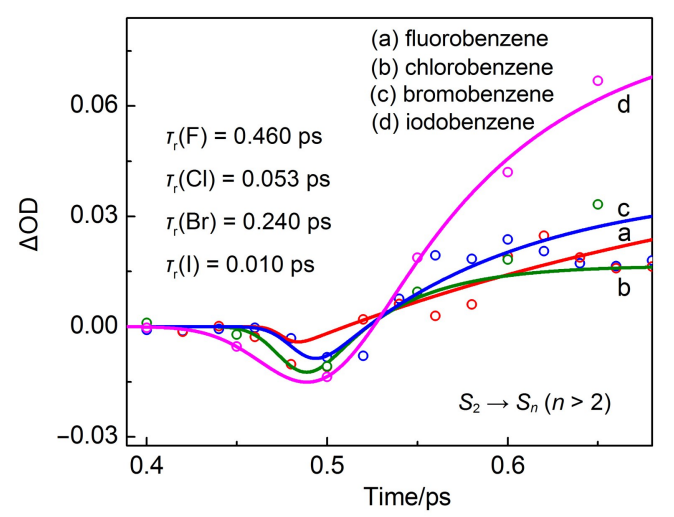

图9 卤代苯溶剂中 3-Ga瞬态吸收 $S_{2}$ 上升沿动力学曲线

Fig.9 Transient absorption $S_{2}$ rise kinetics of 3-Ga at rise curve in halogenated-benzene solvents

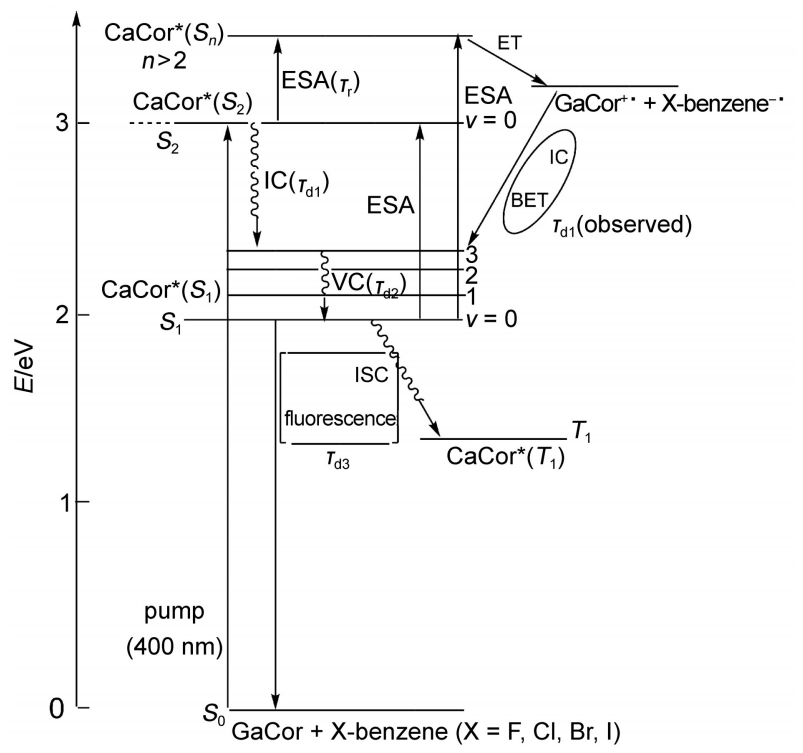

图 10 卤代苯溶剂中 3-Ga激发态可能的弛豫途径图

Fig.10 Possible relaxation pathways of excited state for 3-Ga in halogenated-benzene solvents

BET: back electron transfer; ESA: excited-state absorption; IC: internal conversion; VC: vibration cooling; ISC: intersystem crossing
重组 $S_{2 \mathrm{CS}} \rightarrow S_{2}$ 和内转换过程 $S_{2} \rightarrow S_{1}{ }^{*}$ 。由于 3-Ga 在苯溶剂中 $S_{2} \rightarrow S_{1}{ }^{*}$ 的速度很快 $\left(\tau_{\mathrm{d} 1}=0.28 \mathrm{ps}\right)^{23}$, 可 以近似认为在卤代苯溶剂中的 $\tau_{\mathrm{d} 1}$ 反映了 $3-\mathrm{Ga}$ 的 $S_{2 \mathrm{CS}} \rightarrow S_{2}$ 态电荷重组的速率。据此, 在氟苯、氯 苯, 溴苯和碘苯中 $3-G a$ 的 $S_{2 \mathrm{cs}}$ 态电荷重组的速率常 数 $K_{\mathrm{BET}}\left(\mathrm{BET}\right.$, 反向电子转移) 分别为 $13.0 \times 10^{11}$, $4.07 \times 10^{11}, 3.33 \times 10^{11}$ 和 $1.16 \times 10^{11} \mathrm{~s}^{-1}$ 。可见, 随 着卤代苯溶剂中卤素原子量的增大, $3-\mathrm{Ga}$ 与溶剂 分子形成的 $S_{2 \mathrm{CS}}$ 态电荷重组速度减慢, 而对 $S_{1}{ }^{*} \rightarrow S_{1}$ 振动弛豫的影响则取决溶剂本身的性质, 与重原 子效应无关。需要注意的是, 不同卤代苯溶剂对 3-Ga 的 $S_{1}$ 态物种寿命影响不大, 尽管对苂光寿命 有很大影响, 这是由于 $\tau_{\mathrm{d} 3}$ 反映的是苂光和系间串 越的总和。3-Ga在卤代溶剂中激发态吸收信号的 上升沿动力学曲线见图 9, 单指数拟合得到 $\tau_{\mathrm{r}}$, 反 映的是 $S_{2} \rightarrow S_{n}(n=3,4, \cdots)$ 过程, $\tau_{\mathrm{r}}$ 的数值在 $0.010-0.460 \mathrm{ps}$, 表明它是个超快过程, 溶剂影响 不大。3-Ga激发态可能的弛豫途径归纳如图 10 所 示。

\section{4 结 论}

本文研究了不同卤代苯溶剂对于三种不同氟 原子数取代的镓咔咯光物理性质的影响。卤代苯 溶剂对于镓咔咯紫外光谱的影响主要来自色散力 的作用; 稳态苂光谱图及时间分辨分析结果说 明, 外重原子效应可加速 $S_{1} \rightarrow T_{1}$ 系间窝越速率造 成相应的苂光量子产率的减少; 飞秒瞬态吸收谱 分析结果说明, 在 Soret 带光激发下 3-Ga 与卤代苯 溶剂之间存在光诱导电子转移反应, 形成电荷分 离复合物, 溶剂的重原子效应能减缓其电荷重组 速率, 而对于 $S_{1}$ 态寿命以及 $S_{2} \rightarrow S_{n}(n=3,4, \cdots)$ 过 程影响不大。

\section{References}

(1) Zdilla, M. J.; Abu, O. M. Inorg. Chem. 2008, 47 (22), 10718. doi: $10.1021 /$ ic $801182 \mathrm{q}$

(2) Flamigni, L.; GryKo, D. T. Chem. Soc. Rev. 2009, 38, 1635. doi: $10.1039 / \mathrm{b} 805230 \mathrm{c}$

(3) Gross, Z.; Galili, N.; Saltsman, I. Angew. Chem. Int. Edit. 1999, 38, 1427. doi: 1433-7851/99/3810-1428

(4) Paolesse,R.; Jaquinod, L.; Nurco, D.; Mini, S.; Sagone, F.; Boschi, T.; Smith, K. M. Chem. Commun. 1999, 1307. doi: 10.1039/A903247I

(5) Bose, S.; Pariyar, A.; Biswas, A. N.; Bandyopadhyay, P. Catal. Commum. 2011, 12, 1193. doi: 10.1016/j.catcom.2011.04.026 
(6) Liu, H. Y.; Mahmood, M. H. R.; Qiu, S. X.; Chang, C. K. Coord. Chem. Rev. 2013, 25, 1306. doi: 10.1016/j. ccr.2012.12.017

(7) Chang, C. K.; Kong, P. W.; Liu, H. Y.; Yeung, L. L.; Koon, H. K.; Mak, N. K. Proc. SPIE-Int. Soc. Opt. Eng. 2006, 6139, 613911. doi: 10.1117/12.646328

(8) Zhang, Y.; Chen, H.; Wen, J. Y.; Wang, X. L.; Wang, H.; Ji, L. N.; Liu, H. Y. Chem. J. Chin. Univ. 2013, 34, 2462. [张 阳, 陈 欢, 闻金燕, 王湘利, 王 惠, 计亮年, 刘海洋. 高等化学 学报, 2013, 34, 2462.] doi: 10.7503/cjcu20130610

(9) Robert, C.; Ohkawara, T. Chem. Eur. J. 2014, 20 (16), 4789. doi: $10.1002 /$ chem.v20.16

(10) Zou, H. B.; Yang, H.; Liu, Z. Y.; Liu, H. Y. Organomeallic 2015, 34 (12), 2791. doi: 10.1021/acs.organomet.5b00069

(11) Barata, J. F. B.; Zamarron, A.; Neves, M.; Graca, P. M. S. Eur. J. Med. Chem. 2015, 92, 135. doi: 10.1016/j. ejmech.2014.12.025

(12) Chun, Z.; Jin, X. J. Power Sources 2015, 283, 343. doi: 10.1016/j.jpowsour.2015.02.136

(13) Basumatary, B.; Sekhar, A. Inorg. Chem. 2015, 54 (9), 4257. doi: $10.1021 /$ ic502919s

(14) Santtos, C. I. M.; Oliveria, E. Inorg. Chim. Acta 2014, 417, 148. doi: 10.1016/j.ica.2013.09.049

(15) Agadjannian, H.; Ma, J.; Rentsendorj, A.; Valluripalli, V.; Hwang, J. Y.; Mahammed, A.; Farkas, D. L.; Gray, H. B.; Gross, Z.; Medina-Kauwe, L. K. Proc. Natl. Acad. Sci. U. S. A. 2009, 106 (15), 6105. doi: 10.1073/pnas.0901531106

(16) Wang, J. Y.; Lubow, I.; Chu, D.; Hwang, J. Y.; Lubow, J.; Chu, D.; Ma, J.; Agadjanian, H.; Sims, J.; Gray, H. B.; Gross, Z.; Farkas, D. L.; Lali, K.; Medina-Kauwe, L. K. Mol. Pharm. 2011, 8, 2233. doi: 10.1021/mp200094w

(17) Lim, P.; Mahammed, A.; OKun, Z.; Saltsman, I.; Gross, Z.; Gray, H. B.; Termini, J. Chem. Res. Toxicol. 2012, 25, 400. doi: $10.1021 / \mathrm{tx} 200452 \mathrm{~W}$

(18) Peng, K. M.; Shao, W. L.; Wang, H. H.; Ying, X.; Wang, H.; Ji, L. N.; Liu, H. Y. Acta Phys. -Chim. Sin. 2011, 27 (1), 199. [彭 开美, 邵文莉, 汪华华, 应 晓, 王 惠, 计亮年, 刘海洋. 物理化学学报, 2011, 27 (1), 199.] doi: 10.3866/PKU.
WHXB20110129

(19) Zhang, L.; Liu, Z. Y.; Zhan, X.; Liu, H. Y. Photochem. Photobiol. Sci. 2015, 14, 953. doi: 10.1039/c5pp00060b

(20) Liu, H. Y.; Lai, T. S.; Yeung, L. L. Org. Lett. 2003, 5, 617. doi: 10.1021/o1027111i

(21) Jozefowicz, M.; Bajorek, M.; Heldt, J. J. Lumin. 2014, 153, 152. doi: 10.1016/j.jlumin.2014.03.019

(22) Strat, G.; Strat, M. Spectros. Lett. 1994, 27, 177. doi: 10.1080/ 00387019408000836

(23) Liu, X.; Mahammed, A.; Tripathy, U.; Gross, Z.; Steer, R. P. Chem. Phys. Lett. 2008, 459, 113. doi: 10.1016/j. cplett.2008.05.038

(24) Shao, W. L. Photophysical Study on Ultrafast Electron Transfer in Biomolecular Systems. Sun-Yat Sen University: Guangzhou, 2012; pp 15-30. [邵文莉. 新型光敏剂 Corrole 的激发态动力学及单线态氧产生过程研究[D]. 广州: 中山大 学, 2012: 15-30.]

(25) Yang, X.; Pan, Z. T.; Ma, Y. J. Analytical. Sci. 2003, 19 (6), 588. doi: 10.3969/j.issn.1006-6144.2003.06.028

(26) Paul, G. S.; Martin, G. J. Mol. Spectrosc. 1969, 3, 1. doi: 10.1016/0022-2852(69)90335-X

(27) You, L. L.; Shen, H.; Shi, L.; Zhang, G. L.; Liu, H. Y.; Wang, H.; Ji, L. N. Scientia Sinica chimica 2010, 40, 224. [游丽莉, 沈 涵, 史 蕾, 张国良, 刘海洋, 王 惠, 计亮年. 中国科 学, 2010, 40, 224.]

(28) Chen, P.; Ge, F. Y. Chemistry Online 2014, 77, 243. [陈 鹏, 葛凤燕. 化学通报, 2014, 77, 243.] doi: 10.14159/j.cnki.04413776.2014.03.008

(29) Guo, C.; Feng, M. B. Chin. J. Lumin. 1987, 8 (2), 92. [郭 础, 冯梅波. 发光学报, 1987, 8 (2), 92.]

(30) Shi, L.; Liu, H, Y.; Shen, H. J. Porphyr. Phthalocya. 2009, 13, 1221. doi: $10.1142 / \mathrm{S} 1088424609001546$

(31) Ghosh, M.; Mora, A. K.; Sukhendu, N, P.; Kumar, P. H.; Bangal, P. R.; Sinha, S. J. Photochem. Photobiol. A 2015, 306, 55. doi: 10.1016/j.jphotochem.2015.03.019

(32) Hayes, R, T.; Walsh, C, J.; Wasielewski, M, R. J. Phys. Chem. $A$ 2004, 108, 2375. doi: 10.1021/jp037176i 